\title{
Comparative analysis of the complete plastid genomes of Mangifera species and gene transfer between plastid and mitochondrial genomes
}

\author{
Yingfeng Niu ${ }^{1}$, Chengwen Gao ${ }^{\text {Corresp., } 2}$, Jin Liu ${ }^{\text {Corresp. } 1}$ \\ ${ }^{1}$ Yunnan Institute of Tropical Crops, Xishuangbanna, China \\ 2 The Affiliated Hospital of Qingdao University, Qingdao, China \\ Corresponding Authors: Chengwen Gao, Jin Liu \\ Email address: gaochengwen6@126.com, liujin06@126.com
}

Mango is an important commercial fruit crop belonging to the genus Mangifera. In this study, we reported and compared four newly sequenced plastid genomes of the genus Mangifera, which showed high similarities in overall size (157,780-157,853 bp), genome structure, gene order, and gene content. Three mutation hotspots (trnG-psbZ, psbD-trnT, and ycf4-cemA) were identified as candidate DNA barcodes for Mangifera. These three DNA barcode candidate sequences have high species identification ability. We also identified 12 large fragments that were transferred from the plastid genome to the mitochondrial genome, and found that the similarity was more than $99 \%$. The total size of the transferred fragment was $35,652 \mathrm{bp}$, accounting for $22.6 \%$ of the plastid genome. Fifteen intact chloroplast genes, four tRNAs and numerous partial genes and intergenic spacer regions were identified. There are many of these genes transferred from mitochondria to the chloroplast in other species genomes. Phylogenetic analysis based on whole plastid genome data provided a high support value, and the interspecies relationships within Mangifera were resolved well. 
1 Comparative Analysis of the Complete plastid Genomes of Mangifera 2 Species and Gene Transfer Between plastid and Mitochondrial Genomes

3

4 Yingfeng $\mathrm{Niu}^{1}$, Chengwen $\mathrm{Gao}^{2 *}, \mathrm{Jin} \mathrm{Liu}^{1 *}$

5 'Yunnan Institute of Tropical Crops, Xishuangbanna, China;

$6 \quad{ }^{2}$ The Affiliated Hospital of Qingdao University, Qingdao, China;

7

8

$9 *$ Correspondence

10 Chengwen Gao, The Affiliated Hospital of Qingdao University, Qingdao, 266000, China. Email:

11 gaochengwen6@126.com

12 Jin Liu, Yunnan Institute of Tropical Crops, Xishuangbanna, 666100, China. Email:

13 liujin06@126.com

14 


\section{Abstract}

16 Mango is an important commercial fruit crop belonging to the genus Mangifera. In this study, we

17 reported and compared four newly sequenced plastid genomes of the genus Mangifera, which 18 showed high similarities in overall size (157,780-157,853 bp), genome structure, gene order, and gene content. Three mutation hotspots (trnG-psbZ, psbD-trnT, and ycf4-cemA) were identified as candidate DNA barcodes for Mangifera. These three DNA barcode candidate sequences have high species identification ability. We also identified 12 large fragments that were transferred from the plastid genome to the mitochondrial genome, and found that the similarity was more than $99 \%$. The total size of the transferred fragment was $35,652 \mathrm{bp}$, accounting for $22.6 \%$ of the plastid genome. Fifteen intact chloroplast genes, four tRNAs and numerous partial genes and intergenic spacer regions were identified. There are many of these genes transferred from mitochondria to the chloroplast in other species genomes. Phylogenetic analysis based on whole plastid genome data provided a high support value, and the interspecies relationships within Mangifera were resolved well.

Key words: Mangifera, Chloroplast genome, DNA barcodes, Gene transfer, Phylogenetic analysis 


\section{Introduction}

Mango is a tall, evergreen tree belonging to the genus Mangifera of the Anacardiaceae family. It is an important tropical fruit (Iquebal et al. 2017; Lora \& Hormaza 2018) that originates in tropical and subtropical regions in Southeast Asia (Dutta et al. 2013; Sherman et al. 2015). Owing to its wide range of cultivation (Bajpai et al. 2016), high nutrient value, pleasing appearance, and unique flavor (Surapaneni et al. 2013), it is widely loved by consumers and has the reputation of being known as the "King of Tropical Fruits" (Khan et al. 2015). Southeast Asian countries have a history of mango cultivation that spans thousands of years (Ravishankar et al. 2013). Mangoes were introduced to Africa, South America, and other continents hundreds of years ago, and several varieties suitable for local cultivation have been developed (Mansour et al. 2014; Sennhenn et al. 2014). There are 69 species of mango in the world that are mainly distributed in tropical and subtropical countries including India, Indonesia, the Malay Peninsula, Thailand, and South China, of which, five species are grown in China, namely M. indica, M. persiciformis, M. longipes, $M$. hiemalis, and M. sylvatica; however, the varieties cultivated in production belong to $M$. indica. Phylogenetic analysis of Mangifera species has been a hot topic of research (Nishiyama et al. 2006; Sankaran et al. 2018), while the whole chloroplast genome sequences can provide more genetic information and higher species resolution ability than other molecular data. However, the chloroplast genomes of most Mangifera plants remain unknown (Azim et al. 2014).

Chloroplasts are special organelles that are involved in photosynthesis and consist of layers of thylakoids. They have their own DNA and can split. The chloroplast genome is conserved and consists of four parts. Two inverted repeat (IR) regions separate the small copy region (SSC) and large copy region (LSC). Currently, with the rapid development of next-generation sequencing (NGS) technology, the entire chloroplast genome has been widely used for phylogenetic analysis. They can provide a large number of variable sites for phylogenetic analysis (Gitzendanner et al. 2018). Thus, the entire chloroplast genome shows the potential to resolve evolutionary relationships and produce highly resolved phylogenetic and genetic diversity, particularly in some complex taxa or at low taxonomic levels, which have unresolved relationships (Hu et al. 2016; 
58 Huang et al. 2020; Xu et al. 2019).

59 In this study, the chloroplast genomes of four Mangifera species were sequenced and 60 compared with $M$. Indica and 21 Sapindales plastids. The objectives of this study were as follows:

61 (1) to comparatively analyze the chloroplast genome structure of five species of Mangifera; (2) to 62 identify highly divergent regions of the chloroplast genomes of Mangifera; (3) to determine the 63 insertion of chloroplast genes into mitochondria; (4) to explore the evolutionary relationship 64 between the genus, Mangifera, and Sapindales. Overall, this study would be helpful to further 65 understand plastid evolution and phylogeny of the genus, Mangifera.

66 


\section{Materials and methods}

\section{Plant material, DNA extraction, and sequencing}

Fresh leaves of four Mangifera species (M. hiemalis, M. persiciformis, M. longipes, and M. sylvatica) were collected from Xishuangbanna Tropical Flowers and Plants Garden, South Yunnan, China, and frozen in liquid nitrogen. Total genomic DNA was extracted from all samples according to CTAB method ( $\mathrm{Li}$ et al. 2013). DNA quality was detected using 1\% agarose gel electrophoresis and samples were stored at $-80^{\circ} \mathrm{C}$ until further use.

About 5-10 $\mu \mathrm{g}$ of total DNA were extracted from each of the Mangifera samples to construct a shotgun library with an average insertion size of 300 bp. Paired-end libraries were constructed with NEBNext ${ }^{\circledR}$ DNA Library Prep Master Mix Set for Illumina according to the manufacturer's recommendation. Illumina HiSeq 2500 system (Illumina, San Diego, CA, USA) was used to sequence DNA samples in the paired-end sequencing mode by Novogene Bioinformatics Technology Co. Ltd (Beijing, China), generating approximately 8.0 Gb of raw data per sample. The plastome depth of coverage was more than $2000 \times$.

\section{Chloroplast genome assembly and annotation}

The Trimmomatic v0.38 was used to filter raw sequencing data (Bolger et al. 2014), and the obtained clean data were de novo assembled using SPAdes v3.61 under different K-mer parameters (Bankevich et al. 2012). The scaffolds that were positively associated with chloroplasts were arranged on the reference chloroplast genome of $M$. indica (NC_035239). Paired-end reads were remapped to consensus assembly and multiple iterations were performed to fill in the gaps in the final consensus sequence using Geneious software v2020.0.4 (Kearse et al. 2012).

Chloroplast genome annotation was performed using GeSeq (https://chlorobox.mpimpgolm.mpg.de/geseq.html) to predict genes encoding proteins, transfer RNA (tRNA), and ribosomal RNA (rRNA), and was adjusted manually as needed (Tillich et al. 2017). We also manually examined the IR junctions of all Mangifera species. A circular diagram of the chloroplast genomes of Mangifera was subsequently drawn using OGDRAW v1.3.1 (Greiner et al. 2019).

\section{Genome comparative analysis and divergent hotspot identification}


95

96

97

98

99

100

101

102

103

104

105

106

107

108

109

110

111

112

113

114

115

MAFFT v7.221 was used to align the chloroplast genome sequences of five Mangifera plants (Katoh \& Standley 2013). Next, DnaSP v6.12 was used to perform a sliding window analysis with the step size of $200 \mathrm{bp}$ and window length of $600 \mathrm{bp}$, to detect the rapidly evolving molecular markers for performing phylogenetic analysis (Librado \& Rozas 2009).

\section{Identification of chloroplast gene insertion in mitochondria}

First, we removed the BLAST hits of genes transferred between chloroplast and mitochondrial genomes by mapping the mitochondrial genome of $M$. indica (GenBank: CM021857) to the plastid genomes. Circos v0.69-9 (Krzywinski et al. 2009) software was used to map the mitochondrial and chloroplast genomes of the Mangifera species as well as gene-transfer fragments.

\section{Phylogenetic analysis}

Phylogenetic analyses were performed for five Mangifera (4 species sequenced here) and 21 Sapindales species, using Arabidopsis thaliana as outgroups. MAFFT 7.221 (Katoh \& Standley 2013) was used to align the chloroplast genome sequences of Sapindales species. We used the following three methods to perform phylogenetic analyses of Mangifera species: Bayesian Inference (BI) with a GTR + I + G model using MrBayes v3.2 (Ronquist et al. 2012), the Markov chain Monte Carlo (MCMC) algorithm was run for 1 million generations and sampled every 100 generations. Maximum Likelihood (ML) using MEGA v7.0 with 1000 bootstrap replicates (Kumar et al. 2016), and Maximum Parsimony (MP) with a heuristic search in PAUP v4.0 with 1,000 random taxon stepwise addition sequences (Rédei 2008). A 50\% majority-rule consensus phylogeny was constructed using 1,000 bootstrap replications. 
117

118

119

120

121

122

123

124

125

126

127

128

129

130

131

132

133

134

135

136

137

138

139

140

141

142

143

\section{Results and discussion}

\section{Basic characteristics of the Mangifera chloroplast genomes}

Raw data (approximately from $7.1 \times 10^{9}$ to $8.3 \times 10^{9} \mathrm{bp}$ ) were obtained from M. hiemalis (MN917208), M. persiciformis (MN917209), M. longipes (MN917210), and M. sylvatica (MN917211). The four newly sequenced Mangifera chloroplast genomes have been presented to the GenBank database.

Characteristics of four newly sequenced and one reported Mangifera chloroplast genomes were investigated. Mangifera chloroplast genome sequence sizes were 157,780 157,853 bp (Figure 1), with the largest and smallest being those of $M$. longipes and $M$. indica, respectively. Mangifera chloroplast genomes are characterized by a typical four-part structure, two IR copies (26354-26379 bp) separating the LSC (86673-86726 bp) and SSC (18347-18369 bp) regions. In addition, the GC content of Mangifera genomes was similar, ranging from 37.88-37.89\%. Five Mangifera chloroplast genomes contained 113 predicted functional genes, including 79 proteincoding genes, four ribosomal RNA (rRNA) genes, and 30 transfer RNA (tRNA) genes (Tables 1 and 2). Furthermore, 15 functional genes, including 4 protein-coding genes, four ribosomal RNA genes, and seven transfer RNA gene replicate in the IR regions of the chloroplast genome. The number, type, and order of genes were found to be very similar among the five Mangifera chloroplast genomes (Jo et al. 2017; Rabah et al. 2017; Zhang et al. 2020). The whole chloroplast genome sequences of four Mangifera species were submitted to GenBank with the accession numbers of MN917208 to MN917211.

The IR/SC connected regions were found nearly identical relative positions in the five Mangifera chloroplast genomes (Figure 2). All LSC-IRb connections were found to be located within the rps 19 gene, resulting in a partial expansion of the IRb region to the rps 19 gene (80-104 $\mathrm{bp)}$. The IRb-SSC boundary was located in the $n d h F$ gene, while the SSC-IRa boundary in the five chloroplast genomes was located in the $y c f 1$ gene.

\section{Comparative Mangifera chloroplast genomes and Divergence Hotspot Regions}

Using the comparative sequence analysis of the five species of Mangifera, we found that the 
plastid genome was quite conservative in the five taxa, although there were a few regions with variations. In general, sequences are conserved in the coding region, and most of the detected variations are in the non-coding region. The results agree with previous reports that non-coding regions showed greater divergence than coding regions, this is possibly caused by coding regions affected by stronger selective pressure (Li et al. 2018). Consistent with similar studies involving other plants, the IR regions appear to be more conservative than the LSC and SSC regions (Fig. 1) (Liang et al. 2019; Song et al. 2019). A search for nucleotide substitutions identified 638 variable sites $(0.40 \%)$ in the five chloroplast genomes, including 489 parsimony-informative sites $(0.31 \%)$, this number is smaller than other genus species (Gao et al. 2020; Nguyen et al. 2020).

To identify hotspots of sequence divergence, the nucleotide diversity (Pi) values within the $600 \mathrm{bp}$ window of the Mangifera chloroplast genomes were calculated (Fig. 3). We found that Pi values varied from $0-0.033$, and the three hypervariable regions $(\mathrm{Pi}>0.02)$ of the five Mangifera chloroplast genomes were $\operatorname{trn} G$-psbZ, $p s b D$-trnT, and $y c f 4$-cemA. The $\operatorname{trn} G$-psbZ region exhibited the highest variability $(7.44 \%)$.

Here, we found an increase in the number of variable sites in the following three specific regions based on the results of pairwise plastid genomic alignment and SNP analysis: trnG-psbZ, $p s b D$-trnT, and $y c f 4$-cemA. Thus, Mangifera species may be detected using these regions as novel candidate fragments. Fig. S1 presents the graphical representation of these results using the ML method. These three DNA barcode candidate sequences have high species identification ability. However, further experiments are required to support this Mangifera plastid sequence data.

\section{Characterization of gene transfer of Mangifera chloroplast genome to mitochondrial genome}

The mitochondrial genome of $M$. indica was obtained from GenBank and was 87,1458 bp in size, approximately 5.5 times that of the chloroplast genome consisting of 94 functional genes. We identified 12 large chloroplast genome fragments in the mitochondrial genome, including genes and intergenomic regions. These fragments ranged from 1522-5400 bp and the sequences were over $99 \%$ consistent. The total length of these fragments was $35,652 \mathrm{bp}$, accounting for $22.6 \%$ of the chloroplast genome (Fig. 4 and Table S1). Fifteen intact chloroplast genes (rps 19, rpl2, rpl23, 
171

172

173

174

175

176

177

178

179

180

181

182

183

184

185

186

187

188

189

190

191

192

193

194

195

196

197

$p e t N, r b c L, a c c D, p s b J, p s b L, p s b F, p s b E$, petL, petG, psaA, atpA, cemA ), four tRNAs (trnI-CAU, $\operatorname{trn} C-G C A$, trn $W-C C A$, $\operatorname{trn} P-U G G)$ and numerous partial genes and intergenic spacer regions were identified. There are many of these genes transferred from mitochondria to the chloroplast in other species genomes, such as $r p s 12, r p l 23, r b c L$, petL, petG, trn $W-C C A$ and $\operatorname{trn} P-U G G$ (Gao et al. 2020; Gui et al. 2016).

Intracellular gene transfer exists between different genomes, including those of the chloroplasts, mitochondria, and nuclei (Nguyen et al. 2020; Timmis et al. 2004). Research shows that the frequency of nuclear DNA transfer from organelles in angiosperms is very high (HazkaniCovo et al. 2010; Park et al. 2014; Smith 2011). Gene transfer from chloroplast to mitochondrial genomes is a common phenomenon during long-term evolution (Gui et al. 2016; Nguyen et al. 2020). Due to high sequence identity between the transferred chloroplast genome fragments in the mitochondrial and original chloroplast genomes, gene transfer can lead to assembly errors in these genomes.

\section{Phylogenetic relationship of chloroplast genomes}

In this study, the chloroplast genome was used for infer the phylogenetic location of Mangifera in Sapindales (Fig. 5) and performed a phylogenetic analysis of the chloroplast genome using three different methods, namely, ML, MP, and BI. BI and ML analyses revealed almost the same topology, and most branches had very high support (Fig. S2). However, MP trees differed slightly from BI and ML trees in some taxa (Fig. S3). Despite differences between these three approaches, the relationships between most groups were well resolved and highly supported, suggesting that the use of chloroplast genome data does significantly improve the resolution of phylogenetic analysis. Previous studies have revealed the genetic relationship of Mangifera through morphological, nuclear, amplified fragment length polymorphism, ribosomal internal transcribed spacer (ITS), and partial chloroplast gene analysis (Eiadthong et al. 2000; Nishiyama et al. 2006; Sankaran et al. 2018; Yonemori et al. 2002). The whole chloroplast genome sequencebased phylogenetic tree was built to explore the evolutionary similarities/differences between Mangifera species and between genera in the Sapindales. Phylogenetic analysis based on complete 
198 genome sequences, rather than a few genes, has been carried out in a large number of higher plant 199 species, significantly improving the resolution of phylogenetic analysis (Zhai et al. 2019). 
201

202

203

204

205

206

207

208

209

210

211

212

213

214

215

216

217

218

219

220

221

222

\section{Conclusions}

In this study, the chloroplast genomes of four Mangifera species were sequenced and compared. It was found that the size, structure, and gene content of the Mangifera chloroplast genomes were conserved. Comparative analysis showed a low degree of sequence variation. We identified 13 large fragments that were transferred from the chloroplast genome to the mitochondrial genome. In addition, we identified three mutation hotspots as DNA barcodes for the identification of Mangifera species. These complete chloroplast genome sequences and highly variable markers provide sufficient genetic information for the phylogenetic reconstruction and species identification of the genus Mangifera.

\section{Authors' contributions}

Yingfeng Niu and Jin Liu conceived of the study, wrote and revised the manuscript. Chengwen Gao performed the data analyses, and drafted the earlier version of manuscript. All authors read and approved the final manuscript.

\section{Acknowledgments}

We are grateful to thank Zhangguang Ni for the collection of experiment material.

\section{Data availability statement}

The data that support the findings of this study are openly available in GenBank of NCBI at https:/www.ncbi.nlm.nih.gov, accession number MN917208-MN917211.

\section{Funding information}

This work was supported by Youth Talent Growth Fund of YITC(QNCZ2020-3), Technology Innovation Talents Project of Yunnan Province (2018HB086), Sci-tech Innovation System Construction for Tropical Crops Grant of Yunnan Province (No.RF2020-9) 
224

225

226

227

228

229

230

231

232

233

234

235

236

237

238

239

240

241

242

243

244

245

246

247

248

249

250

251

252

253

254

255

256

257

258

259

260

261

262

263

\section{References}

Azim, M.K., Khan, I.A. \& Zhang, Y. 2014. Characterization of mango (Mangifera indica L.) transcriptome and chloroplast genome. Plant Molecular Biology 85:193-208. 10.1007/s11103-014-0179-8

Bajpai A, Muthukumar M, Ahmad I, Ravishankar KV, Parthasarthy VA, Sthapit B, Rao R, Verma SP, and Rajan S. 2016. Molecular and morphological diversity in locally grown non-commercial (heirloom) mango varieties of North India. Journal of Environmental Biology 37:221-228.

Bankevich A, Nurk S, Antipov D, Gurevich AA, Dvorkin M, Kulikov AS, Lesin VM, Nikolenko SI, Pham S, Prjibelski AD, Pyshkin AV, Sirotkin AV, Vyahhi N, Tesler G, Alekseyev MA, and Pevzner PA. 2012. SPAdes: A New Genome Assembly Algorithm and Its Applications to Single-Cell Sequencing. Journal of Computational Biology 19:455-477. 10.1089/cmb.2012.0021

Bolger AM, Lohse M, and Usadel B. 2014. Trimmomatic: a flexible trimmer for Illumina sequence data. Bioinformatics 30:2114-2120. 10.1093/bioinformatics/btu170

Dutta SK, Srivastav M, Rymbai H, Chaudhary R, Singh AK, Dubey AK, and Lal K. 2013. Pollen-pistil interaction studies in mango (Mangifera indica L.) cultivars. Scientia Horticulturae 160:213-221. 10.1016/j.scienta.2013.05.012

Eiadthong W, Yonemori K, Kanzaki S, Sugiura A, Utsunomiya N, and Subhadrabandhu S. 2000. Amplified fragment length polymorphism analysis for studying genetic relationships among Mangifera species in Thailand. Journal of the American Society for Horticultural Science 125:160-164. 10.21273/jashs.125.2.160

Gitzendanner MA, Soltis PS, Wong GKS, Ruhfel BR, and Soltis DE. 2018. Plastid phylogenomic analysis of green plants: A billion years of evolutionary history. American Journal of Botany 105:291-301. 10.1002/ajb2.1048

Greiner S, Lehwark P, and Bock R. 2019. OrganellarGenomeDRAW (OGDRAW) version 1.3.1: expanded toolkit for the graphical visualization of organellar genomes. Nucleic Acids Research 47:W59-W64. 10.1093/nar/gkz238

Gao CW, Wu CH, ZhangQ, Zhao X, Wu MX, Chen RR, ZhaoYL and Li ZQ. 2020. Characterization of chloroplast genomes from two Salvia medicinal plants and gene transfer among their mitochondrial and chloroplast genomes. Frontiers in Genetics 10.3389/fgene.2020.574962

Gui ST, Wu ZH, Zhang HY, Zheng YZ, Zhu ZX, Liang DQ, and Ding Y. 2016. The mitochondrial genome map of Nelumbo nucifera reveals ancient evolutionary features. Scientific Reports 6:11. 10.1038/srep30158

Hazkani-Covo E, Zeller RM, and Martin W. 2010. Molecular Poltergeists: Mitochondrial DNA Copies (numts) in Sequenced Nuclear Genomes. Plos Genetics 6:11. 10.1371/journal.pgen.1000834

Hu H, Hu QJ, Al-Shehbaz IA, Luo X, Zeng TT, Guo XY, and Liu JQ. 2016. Species Delimitation and Interspecific Relationships of the Genus Orychophragmus (Brassicaceae) Inferred from Whole Chloroplast Genomes. Frontiers in Plant Science 7:10. 10.3389/fpls.2016.01826

Huang J, Yu Y, Liu YM, Xie DF, He XJ, and Zhou SD. 2020. Comparative Chloroplast Genomics of Fritillaria (Liliaceae), Inferences for Phylogenetic Relationships between Fritillaria and Lilium and Plastome Evolution. Plants-Basel 9:15. 10.3390/plants9020133

Iquebal MA, Jaiswal S, Mahato AK, Jayaswal PK, Angadi UB, Kumar N, Sharma N, Singh AK, Srivastav M, Prakash J, Singh SK, Khan K, Mishra RK, Rajan S, Bajpai A, Sandhya BS, Nischita P, Ravishankar KV, 
Dinesh MR, Rai A, Kumar D, Sharma TR, and Singh NK. 2017. MiSNPDb: a web-based genomic resources of tropical ecology fruit mango (Mangifera indica L.) for phylogeography and varietal differentiation. Scientific Reports 7:9. 10.1038/s41598-017-14998-2

Jo S, Kim HW, Kim YK, Sohn JY, Cheon SH, and Kim KJ. 2017. The complete plastome sequences of Mangifera indica L. (Anacardiaceae). Mitochondrial DNA Part B-Resources 2:698-700. 10.1080/23802359.2017.1390407

Katoh K, and Standley DM. 2013. MAFFT Multiple Sequence Alignment Software Version 7: Improvements in Performance and Usability. Molecular Biology and Evolution 30:772-780. 10.1093/molbev/mst010

Kearse M, Moir R, Wilson A, Stones-Havas S, Cheung M, Sturrock S, Buxton S, Cooper A, Markowitz S, Duran C, Thierer T, Ashton B, Meintjes P, and Drummond A. 2012. Geneious Basic: An integrated and extendable desktop software platform for the organization and analysis of sequence data. Bioinformatics 28:16471649. 10.1093/bioinformatics/bts199

Khan AS, Ali S, and Khan IA. 2015. Morphological and molecular characterization and evaluation of mango germplasm: An overview. Scientia Horticulturae 194:353-366. 10.1016/j.scienta.2015.08.031

Krzywinski M, Schein J, Birol I, Connors J, Gascoyne R, Horsman D, Jones SJ, and Marra MA. 2009. Circos: An information aesthetic for comparative genomics. Genome Research 19:1639-1645. 10.1101/gr.092759.109

Kumar S, Stecher G, and Tamura K. 2016. MEGA7: Molecular Evolutionary Genetics Analysis Version 7.0 for Bigger Datasets. Molecular Biology and Evolution 33:1870-1874. 10.1093/molbev/msw054

Li J, Wang S, Jing Y, Ling W, and Zhou S. 2013. A modified CTAB protocol for plant DNA extraction. Chinese Bulletin of Botany.

Li YT, Zhang J, Li LF, Gao LJ, Xu JT, and Yang MS. 2018. Structural and Comparative Analysis of the Complete Chloroplast Genome of Pyrus hopeiensis"Wild Plants with a Tiny Population"and Three Other Pyrus Species. International Journal of Molecular Sciences 19:19. 10.3390/ijms19103262

Liang CL, Wang L, Lei J, Duan BZ, Ma WS, Xiao SM, Qi HJ, Wang Z, Liu YQ, Shen XF, Guo S, Hu HY, Xu J, and Chen SL. 2019. A Comparative Analysis of the Chloroplast Genomes of Four Salvia Medicinal Plants. Engineering 5:907-915. 10.1016/j.eng.2019.01.017

Librado P, and Rozas J. 2009. DnaSP v5: a software for comprehensive analysis of DNA polymorphism data. Bioinformatics 25:1451-1452. 10.1093/bioinformatics/btp187

Lora J, and Hormaza JI. 2018. Pollen wall development in mango (Mangifera indica L., Anacardiaceae). Plant Reproduction 31:385-397. 10.1007/s00497-018-0342-5

Mansour H, Mekki LE, and Hussein MA. 2014. Assessment of genetic diversity and relationships among Egyptian mango (Mangifera indica L.) cultivers grown in Suez Canal and Sinai region using RAPD markers. Pakistan journal of biological sciences : PJBS 17:56-61.

Nguyen VB, Giang VNL, Waminal NE, Park HS, Kim NH, Jang W, Lee J, and Yang TJ. 2020. Comprehensive comparative analysis of chloroplast genomes from seven Panax species and development of an authentication system based on species-unique single nucleotide polymorphism markers. Journal of Ginseng Research 44:135-144. 10.1016/j.jgr.2018.06.003

Nishiyama K, Choi YA, Honsho C, Eiadthong W, and Yonemori K. 2006. Application of genomic in situ hybridization for phylogenetic study between Mangifera indica L. and eight wild species of Mangifera. Scientia Horticulturae 110:114-117. 10.1016/j.scienta.2006.06.005

Park S, Ruhlman TA, Sabir JSM, Mutwakil MHZ, Baeshen MN, Sabir MJ, Baeshen NA, and Jansen RK. 2014. 
305

306

307

308

309

310

311

312

313

314

315

316

317

318

319

320

321

322

323

324

325

326

327

328

329

330

331

332

333

334

335

336

337

338

339

340

341

342

343

344

345

Complete sequences of organelle genomes from the medicinal plant Rhazya stricta (Apocynaceae) and contrasting patterns of mitochondrial genome evolution across asterids. Bmc Genomics 15:18.

10.1186/1471-2164-15-405

Rabah SO, Lee C, Hajrah NH, Makki RM, Alharby HF, Alhebshi AM, Sabir JSM, Jansen RK, and Ruhlman TA. 2017. Plastome Sequencing of Ten Nonmodel Crop Species Uncovers a Large Insertion of Mitochondrial DNA in Cashew. Plant Genome 10:14. 10.3835/plantgenome2017.03.0020

Ravishankar KV, Dinesh MR, Mani BH, Padmakar B, and Vasugi C. 2013. Assessment of Genetic Diversity of Mango (Mangifera indica L.) Cultivars from Indian Peninsula Using Sequence Tagged Microsatellite Site (STMS) Markers. In: Lu P, ed. Ix International Mango Symposium. Leuven 1: Int Soc Horticultural Science, 269-275.

Rédei GP. 2008. PAUP (phylogenetic analysis using parsimony).

Ronquist F, Teslenko M, van der Mark P, Ayres DL, Darling A, Hohna S, Larget B, Liu L, Suchard MA, and Huelsenbeck JP. 2012. MrBayes 3.2: Efficient Bayesian Phylogenetic Inference and Model Choice Across a Large Model Space. Systematic Biology 61:539-542. 10.1093/sysbio/sys029

Sankaran M, Dinesh MR, Chaitra N, and Ravishankar KV. 2018. Morphological, cytological, palynological and molecular characterization of certain Mangifera species. Current Science 115:1379-1386. $10.18520 / \mathrm{cs} / \mathrm{v} 115 / \mathrm{i} 7 / 1379-1386$

Sennhenn A, Prinz K, Gebauer J, Whitbread A, Jamnadass R, and Kehlenbeck K. 2014. Identification of mango (Mangifera indica L.) landraces from Eastern and Central Kenya using a morphological and molecular approach. Genetic Resources and Crop Evolution 61:7-22. 10.1007/s10722-013-0012-2

Sherman A, Rubinstein M, Eshed R, Benita M, Ish-Shalom M, Sharabi-Schwager M, Rozen A, Saada D, Cohen Y, and Ophir R. 2015. Mango (Mangifera indica L.) germplasm diversity based on single nucleotide polymorphisms derived from the transcriptome. Bmc Plant Biology 15:11. 10.1186/s12870-015-0663-6

Smith DR. 2011. Extending the Limited Transfer Window Hypothesis to Inter-organelle DNA Migration. Genome Biology and Evolution 3:743-748. 10.1093/gbe/evr068

Song Y, Zhang YJ, Xu J, Li WM, and Li MF. 2019. Characterization of the complete chloroplast genome sequence of Dalbergia species and its phylogenetic implications. Scientific Reports 9:10. 10.1038/s41598-01956727-x

Surapaneni M, Vemireddy LR, Begum H, Reddy BP, Neetasri C, Nagaraju J, Anwar SY, and Siddiq EA. 2013. Population structure and genetic analysis of different utility types of mango (Mangifera indica L.) germplasm of Andhra Pradesh state of India using microsatellite markers. Plant Systematics and Evolution 299:1215-1229. 10.1007/s00606-013-0790-1

Tillich M, Lehwark P, Pellizzer T, Ulbricht-Jones ES, Fischer A, Bock R, and Greiner S. 2017. GeSeq - versatile and accurate annotation of organelle genomes. Nucleic Acids Research 45:W6-W11. 10.1093/nar/gkx391

Timmis JN, Ayliffe MA, Huang CY, and Martin W. 2004. Endosymbiotic gene transfer: Organelle genomes forge eukaryotic chromosomes. Nature Reviews Genetics 5:123-U116. 10.1038/nrg1271

Xu WQ, Losh J, Chen C, Li P, Wang RH, Zhao YP, Qiu YX, and Fu CX. 2019. Comparative genomics of figworts (Scrophularia, Scrophulariaceae), with implications for the evolution of Scrophularia and Lamiales. Journal of Systematics and Evolution 57:55-65. 10.1111/jse.12421

Yonemori K, Honsho C, Kanzaki S, Eiadthong W, and Sugiura A. 2002. Phylogenetic relationships of Mangifera species revealed by ITS sequences of nuclear ribosomal DNA and a possibility of their hybrid origin. Plant

Peer) reviewing PDF | (2020:08:51712:2:0:NEW 18 Dec 2020) 
Systematics and Evolution 231:59-75. 10.1007/s006060200011

347

348

349

350

351

352

353
Zhai W, Duan XS, Zhang R, Guo CC, Li L, Xu GX, Shan HY, Kong HZ, and Ren Y. 2019. Chloroplast genomic data provide new and robust insights into the phylogeny and evolution of the Ranunculaceae. Molecular Phylogenetics and Evolution 135:12-21. 10.1016/j.ympev.2019.02.024

Zhang Y, Ou KW, Huang GD, Lu YF, Yang GQ, and Pang XH. 2020. The complete chloroplast genome sequence of Mangifera sylvatica Roxb. (Anacardiaceae) and its phylogenetic analysis. Mitochondrial DNA Part BResources 5:738-739. 10.1080/23802359.2020.1715286 
354

355

356

357

358

359

360

361

362

363

364

365

366

367

368

369

370

371

372

\section{Figure legends}

Figure 1. Sequence diagram of Mangifera chloroplast genomes. Gene map of Mangifera chloroplast genomes, sequence alignment of Mangifera species chloroplast genome (a: $M$. Sylvatica, b: M. hiemalis, c: M. longipes, d: M. persiciformis with reference to $M$. indica), GC content, and GC skew from the outside to inside.

Figure 2. Comparison of inverted repeat (IR) boundary among Mangifera species, where genes and gene fragments across IRa/b junctions are represented in color boxes above the horizontal line. Genes and IR segments are not mapped to scale.

Figure 3. Mangifera Chloroplast genomes sliding window analysis (window length: $600 \mathrm{bp}$; step size: 200 bp). X-axis: Position of a window; Y-axis: Genetic diversity per window.

Figure 4. Schematic diagram of gene transfer between chloroplast and mitochondria in Mangifera species. Colored lines within the circle show where the chloroplast genome is inserted into the mitochondrial genome. Genes within a circle are transcribed clockwise, while those outside the circle are transcribed counterclockwise.

Figure 5. ML phylogenetic tree of five Mangifera species with 21 related species in the Sapindales based on whole chloroplast genome sequence. Numbers related to the branches are ML bootstrap value, MP bootstrap value, and Bayesian posterior probability, respectively. Asterisk denotes $100 \%$ bootstrap support or 1.0 posterior probability. 


\section{Supporting information}

375 Additional supporting information may be found in the online version of this article.

376 Figure S1. Phylogenetic tree of Mangifera species using maximum likelihood (ML) methods

377 based on three mutation hotspots.

378 Figure S2. Phylogenetic trees of Sapindales based on Bayesian analysis

379 Figure S3. Phylogenetic trees of Sapindales based on maximum parsimony (MP) analysis

380 Figure S4. Morphological characteristics of fruits of five Mangifera species

381 Table S1. Blast results between chloroplast and mitochondrial genome in Mangifera. 
Figure 1

Sequence diagram of Mangifera chloroplast genomes

Gene map of Mangifera chloroplast genomes, sequence alignment of Mangifera species chloroplast genome (a: M. Sylvatica, b: M. hiemalis, c: M. longipes, d: M. persiciformis with reference to $M$. indica), GC content, and GC skew from the outside to inside. 


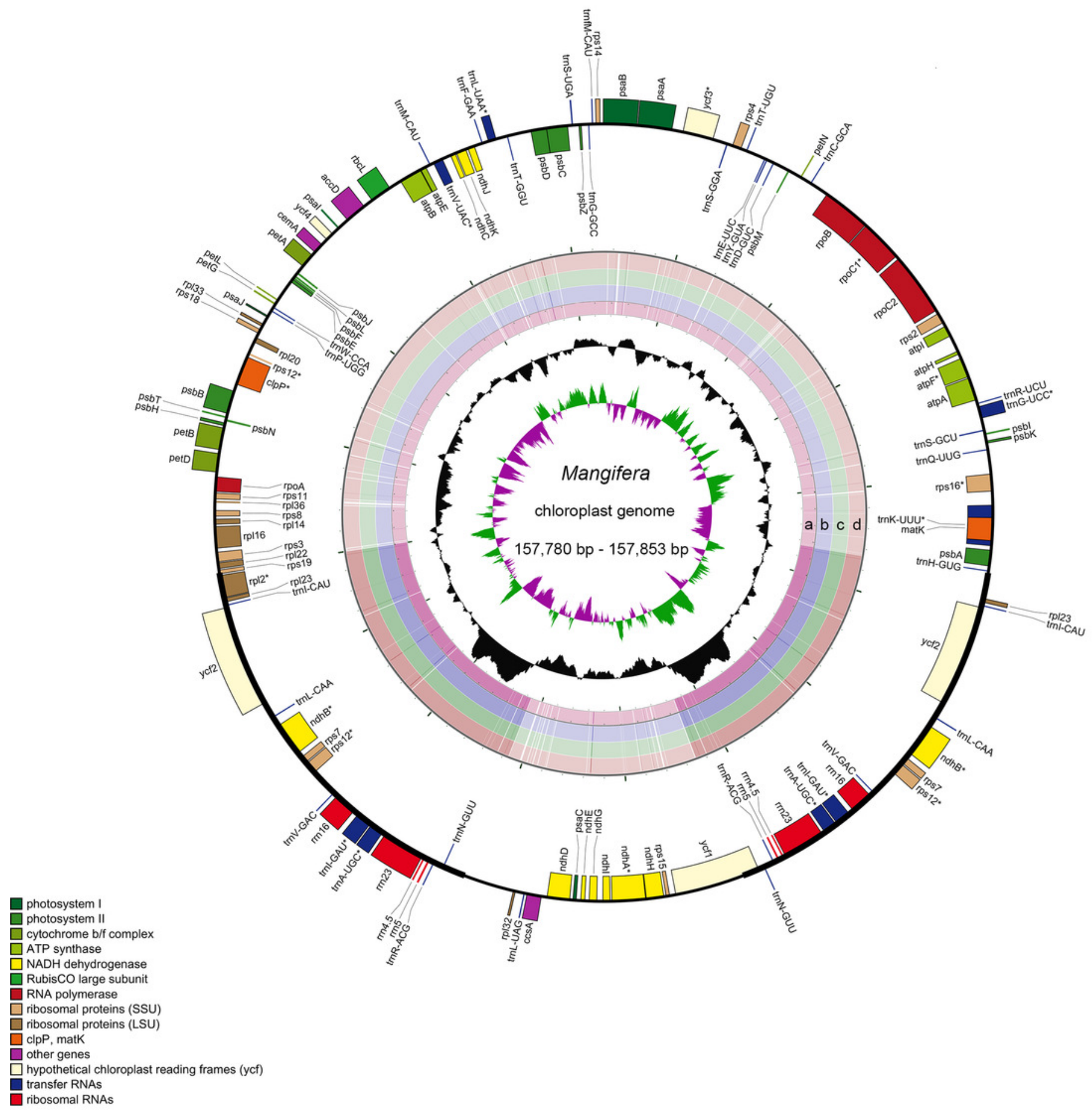




\section{Figure 2}

Comparison of inverted repeat (IR) boundary among Mangifera species

Comparison of inverted repeat (IR) boundary among Mangifera species, where genes and gene fragments across IRa/b junctions are represented in color boxes above the horizontal line. Genes and IR segments are not mapped to scale.

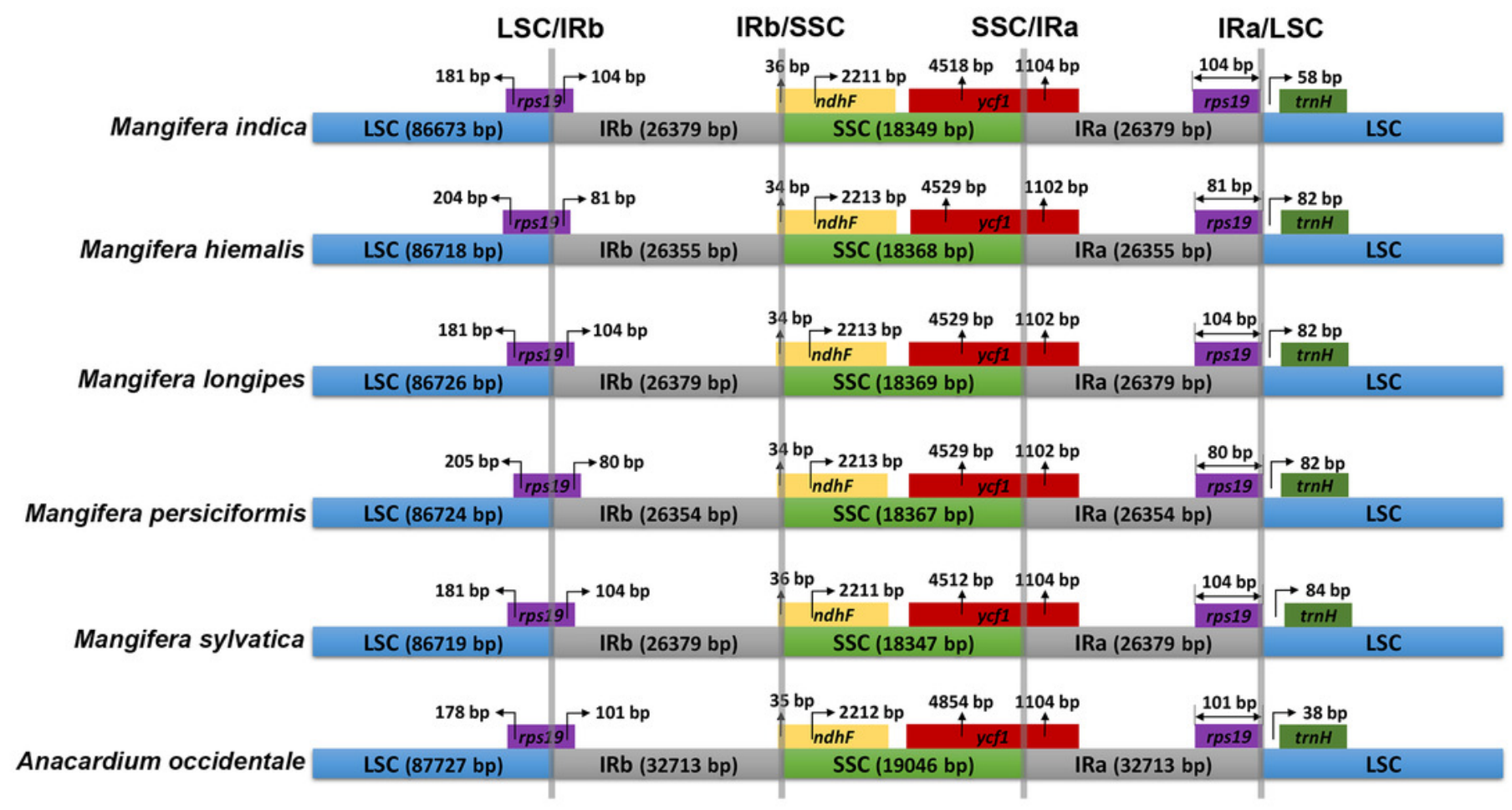


Figure 3

Mangifera Chloroplast genomes sliding window analysis (window length: $600 \mathrm{bp}$; step size: $200 \mathrm{bp})$.

X-axis: Position of a window; Y-axis: Genetic diversity per window.

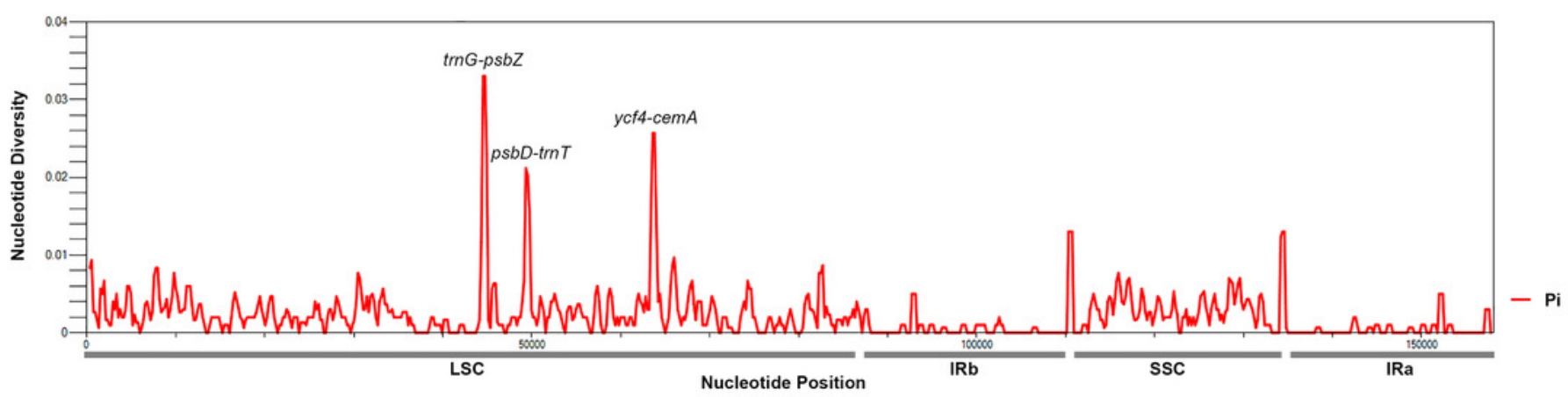




\section{Figure 4}

Schematic diagram of gene transfer between chloroplast and mitochondria in Mangifera species.

Colored lines within the circle show where the chloroplast genome is inserted into the mitochondrial genome. Genes within a circle are transcribed clockwise, while those outside the circle are transcribed counterclockwise. 


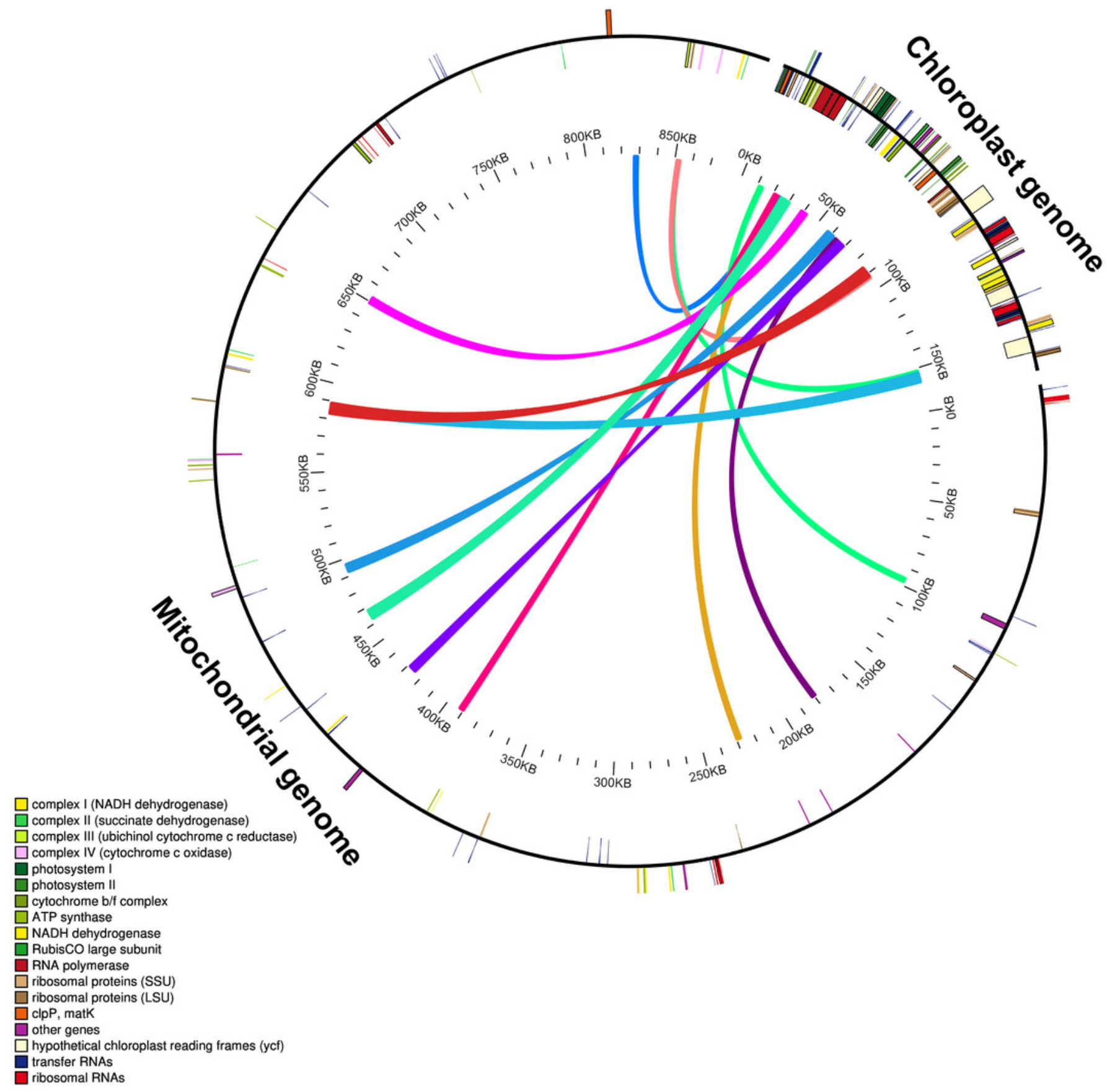


Figure 5

ML phylogenetic tree of five Mangifera species with 21 related species in the Sapindales based on whole chloroplast genome sequence.

Numbers related to the branches are ML bootstrap value, MP bootstrap value, and Bayesian posterior probability, respectively. Asterisk denotes 100\% bootstrap support or 1.0 posterior probability.

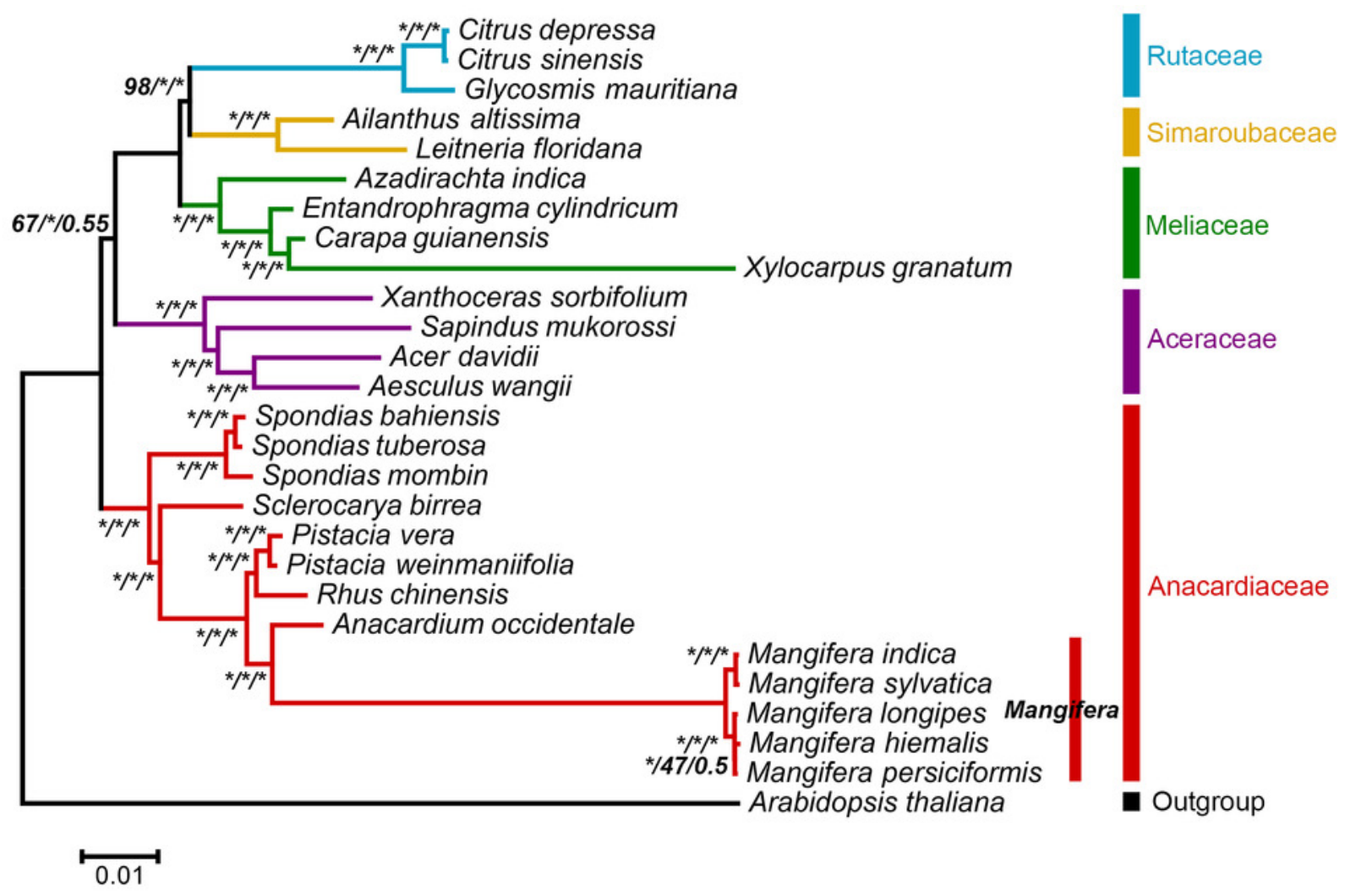




\section{Table $\mathbf{1}$ (on next page)}

Summary of chloroplast genome features of five Mangifera species

Summary of chloroplast genome features of five Mangifera species 
1 Table 1 - Summary of chloroplast genome features of five Mangifera species.

\begin{tabular}{llllll}
\hline Genome feature & M. indica & M. longipes & $\begin{array}{l}\text { M. } \\
\text { persiciformis }\end{array}$ & $\begin{array}{l}\text { M. } \\
\text { hiemalis }\end{array}$ & M. sylvatica \\
\hline Total size (bp) & 157,780 & 157,853 & 157,799 & 157,796 & 157,824 \\
LSC Length (bp) & 86,673 & 86,726 & 86,724 & 86,718 & 86,719 \\
SSC Length (bp) & 18,349 & 18,369 & 18,367 & 18,368 & 18,347 \\
IR Length (bp) & 26,379 & 26,379 & 26,354 & 26,355 & 26,379 \\
Total Genes & 113 & 113 & 113 & 113 & 113 \\
Protein coding Genes & 79 & 79 & 79 & 79 & 79 \\
Structure RNAs & 34 & 34 & 34 & 34 & 34 \\
GC Content (\%) & $37.89 \%$ & $37.88 \%$ & $37.88 \%$ & $37.89 \%$ & $37.89 \%$ \\
GenBank Accessions & NC035239 & MN917210 & MN917209 & MN917208 & MN917211 \\
\hline
\end{tabular}


Table 2 (on next page)

Genes contained in Mangifera chloroplast genome

Genes contained in Mangifera chloroplast genome 
1 Table 2 - Genes contained in Mangifera chloroplast genome.

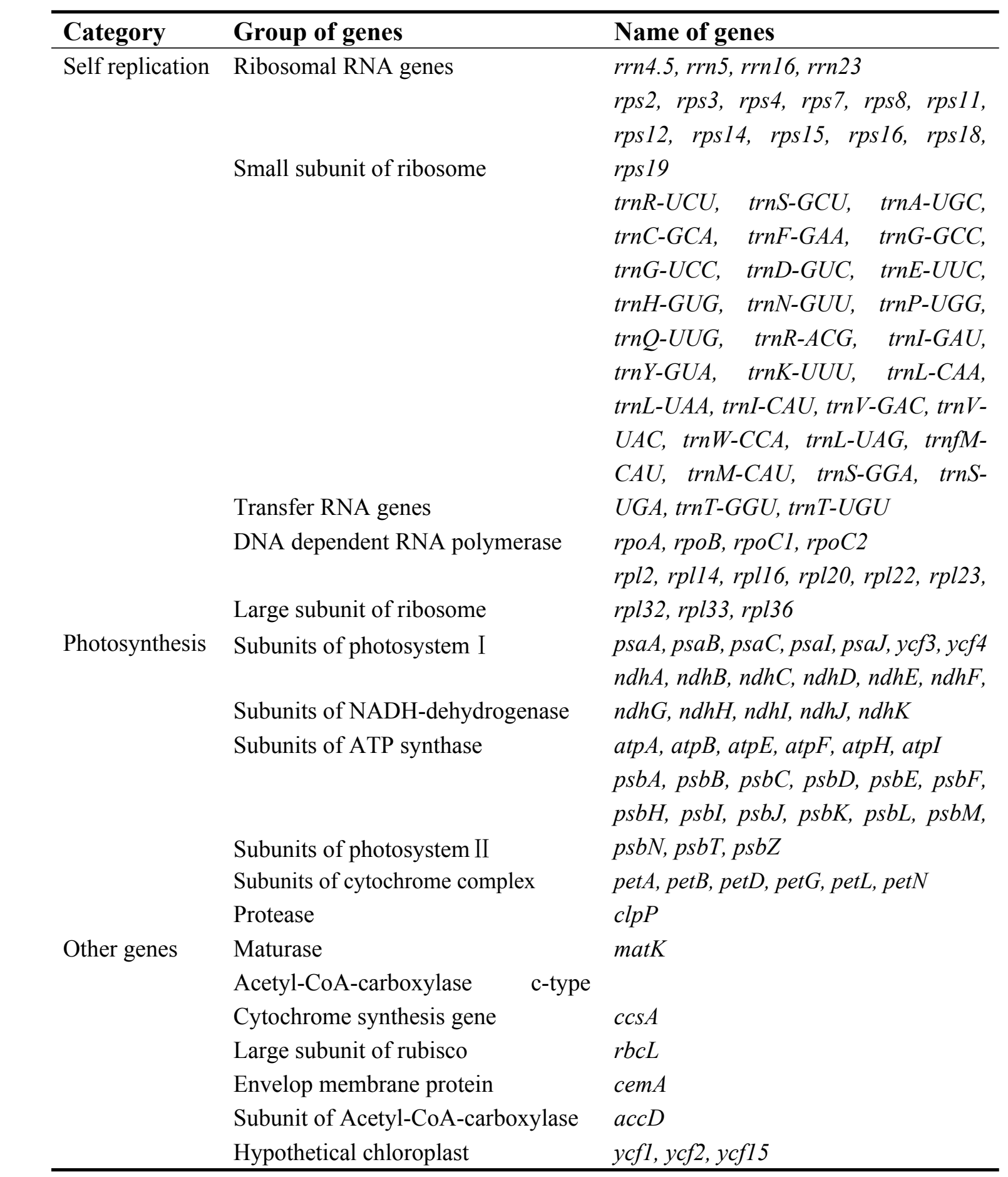

